\title{
Coarse - grained molecular dynamics simulation of cross - linking of DGEBA epoxy resin and estimation of the adhesive strength
}

\author{
A.V.S. Siva Prasad, Tarun Grover, Sumit Basu*
}

Department of Mechanical Engineering, Indian Institute of Technology, Kanpur, INDIA 208016

"Corresponding Author: e-mail: sbasu@iitk.ac.in

\begin{abstract}
In this work we attempt to predict the work of separation of crosslinked diglycidyl ether of bisphenol (A) (DGEBA) adhesive confined between two rigid adherends. To this end we start from merely the molecular structure of DGEBA and coarse grain the molecule into a chain comprised of three superatoms. These three superatom structures are then allowed to crosslink (based on the attainment of a minimum distance of approach) with a coarse grained crosslinker molecule in a Molecular Dynamics (MD) simulation. The crosslinked samples, confined between rigid walls and subjected to slab boundary conditions are pulled apart at a high rate of strain to complete separation. The energy expended in causing complete separation is compared with Linear Elastic Fracture Mechanics estimates and experimental determinations of the work of separation. Our predictions of the value of the work of separation, obtained from only a description of the molecular architecture of DGEBA and the crosslinker molecule, falls within the range of scatter in the experimental measurements.
\end{abstract}

Keywords: Molecular Dynamics, Coarse - Graining, Cross - linking, Adhesion Energy

\section{Introduction}

Predicting macroscopic properties of a polymer starting from a description of its molecular architecture is a long standing scientific goal. The problem however, involves a large variety of length and time scales. The range of length scales span a wide spectrum from $0.1 \mathrm{~nm}$ which is a typical bond length, to the scale at which typical mechanical tests are conducted i.e. tens of millimeters. Kuhn lengths for typical polymers are of the order of $1 \mathrm{~nm}$ for randomly coiled chains and typical radii of gyration of polymer chains at realistic molecular weights are of the order of $10 \mathrm{~nm}$. Time scales too range from $1 \mathrm{fs}$, the vibration time period for individual bonds to several seconds, the typical relaxation times for commercial polymers. The fact that the structure and dynamics is governed by such a wide spectrum of time and length scales makes multiscale simulation of these materials especially difficult (Theodorou, 2004).

Atomistically detailed simulations with a view to obtaining macroscopic and in particular, mechanical properties of macromolecular systems have been conducted by several researchers. These include, among others, simulations of stress strain response of glassy polymers by Brown and Clarke (1991), Ogura and Yamomato (1995), Capaldi et al. (2002), Yashiro et al. (2003), Negi and Basu (2006), Lyulin and Michels (2006); viscoelastic properties by Weiner and Gao (1994); adhesive properties by Stevens (2001), Rottler and Robbins (2003) and Kulmi and Basu (2006).

The simulation studies mentioned above use force field models of basically two types. A large body of literature exists on polyethylene (PE)-like models where a 'united atom' represents a $-\mathrm{CH}_{2}$ - unit each of which interacts with bond stretching, bending and torsions potential with four of its bonded neighbours and through the Lenard Jones (LJ) potential with the rest. The parameters for the force fields are obtained from ab initio calculations on n-butane as in typical calculations by Steele (1985). Similar models obtained by absorbing the hydrogens into united atoms have been proposed for more complicated molecules like polystyrene (see, Mondello et al. 1994) and used in MD studies to predict mechanical properties by Lyulin and Michels (2006). Some investigators have used the 'bead spring model' following Kremer and Grest (1995) that uses a finite extensible non linear spring (FENE) bond potential preventing crossing of chains in conjunction with a purely repulsive LJ potential of non bonded interactions. This produces flexible macromolecular chains which can rotationally be stiffened by the addition of bending and torsion potentials (see, Bulacu and van der Giessen, 2005). Both models, in order to be used for a particular macromolecular 
system need to use parameters that are obtained from lower level simulations.

A related and somewhat more realistic approach is to coarse grain a particular macromolecule such that groups of atoms in one or more monomers constituting it are systematically mapped onto superatoms, thus reducing significantly the total number of degrees of freedom that need to be accounted for. If efficient strategies for quantifying the interaction between the superatoms can be devised, the process can probably be automated and more importantly, lead to very significant improvements in the time and length scales accessible to MD simulations performed with the coarse grained systems over those with all-atoms models. Examples of such attempts to coarse grain particular systems include those by Tschop et al. (1998), Akkermans and Briels (2001), Baschnagel et al. (2000), Muller-Plathe (2002) and the works by Faller and co-workers, especially Sun and Faller (2005). The coarse grained macromolecules are basically linear chains comprised of superatoms interacting through the derived force fields. Ensembles of such chains can then be used in MD simulations with a view to obtaining useful mechanical or other properties. The properties thus derived are essentially 'parameter-free', 'first principle' estimates.

Our objective in the present paper is to obtain the adhesive strength of a common commercial adhesive using such a 'parameterfree' procedure and compare with published experimental data. As a test case to this end, we have chosen the commonly used adhesive DGEBA with diamine as crosslinker. Properties of this adhesive system like its temperature dependent density, glass transition temperature and strength estimates from various adhesion tests are well known. For example, Fujii et al (1996) have conducted several experiments on a number of configurations of adherends to estimate the adhesive strength of this adhesive. These results therefore, give us an effective benchmark against which our estimates can be compared.
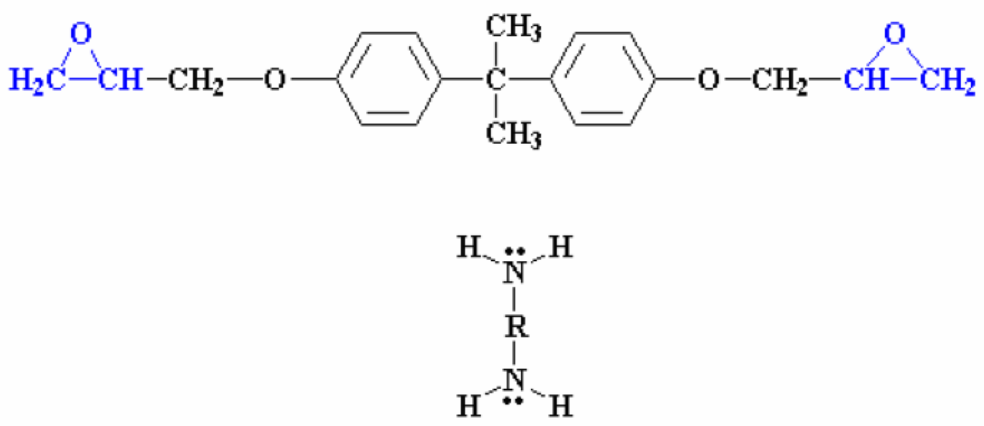

(a)
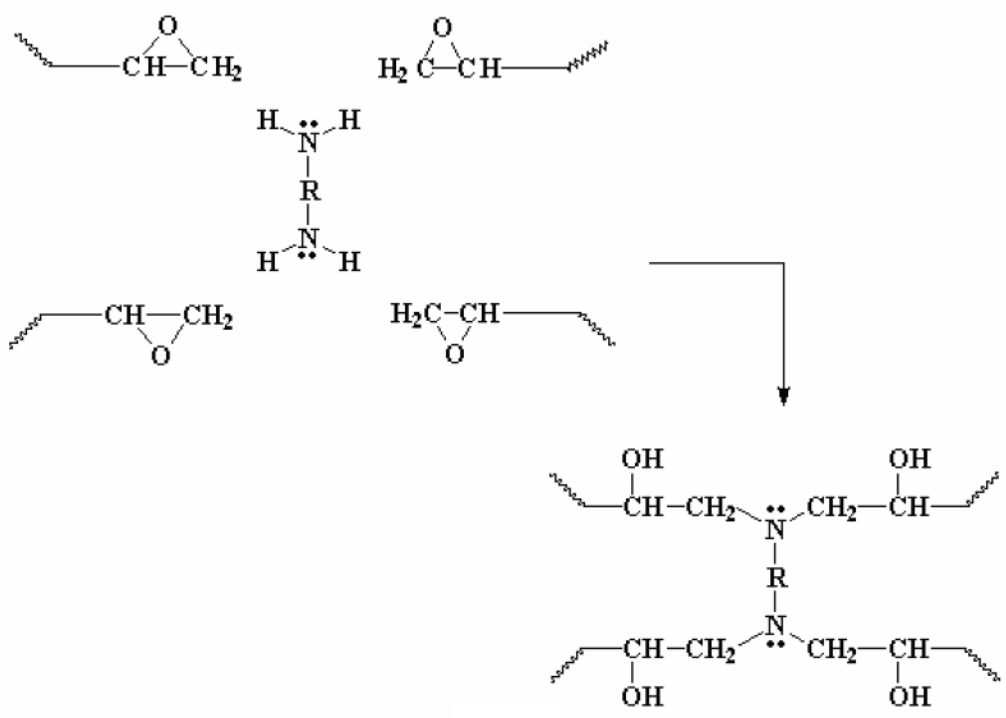

(b)

Figure 1: Figure showing (a) Molecular formulae of DGEBA monomer and diamine crosslinker and (b) the crosslinking mechanism. The symbol R stands for $-\mathrm{CH}_{2}-\mathrm{CH}_{2}-\mathrm{NH}-\mathrm{CH}_{2}-\mathrm{CH}_{2}-\mathrm{NH}-\mathrm{CH}_{2}-\mathrm{CH}_{2}-$. 
The molecular structure of DGEBA and the diamine crosslinker is shown in Fig. 1(a). The reaction between the DGEBA and the crosslinker takes place in the manner shown in Fig. 1(b). It should be noted from Fig. 1(b) that at most four DGEBA molecules can be attached to a diamine during crosslinking. The steps to be adopted in this work to determine the adhesive energy are as follows. We will start with the DGEBA molecule and find its optimum structure. A coarse graining procedure similar to Tschop et al. (1998) is followed whereby each DGEBA molecule containing tens of atoms are reduced to an effective molecule having only 3 superatoms. The interactions between these superatoms are also characterized in the manner prescribed by Tschop et al. (1998). The crosslinker atom is also trivially coarse grained into a single superatom. This is followed by a crosslinking simulation on the coarse grained DGEBA and crosslinker whence a crosslinked network of DGEBA is formed between two rigid adherends. The adherends connected by the crosslinked adhesive are then pulled apart till they separate and the energy of separation is computed. Relevant fracture mechanics estimates of the energy of separation are then compared with the energy obtained from the coarse grained MD.

The organisation of the paper follows the methodology outlined above. The next section explains the coarse graining procedure as applied to DGEBA. In Sec. 3 we discuss the crosslinking simulation and the samples generated. Results from pulling the networked sample apart and the review of fracture mechanics concepts are discussed in Sec. 4 . The salient features of this work are summarized in Sec. 5.

\section{Coarse graining of DGEBA}

In Tschop et al. (1998), as well as in McCoy and Curro (1998) and Akkermans and Briels (2001), coarse graining was done on melts such as Bisphenol-A-Polycarbonate. We apply the same procedure as outline in Tschop et al. (1998) to DGEBA.

The basic requirement of the procedure is that both the all atom system as well as the coarse grained system should, under similar circumstances, yield the same average value for a given macroscopic quantity $F$. In other words, if $x_{1}, x_{2}, x_{3}, \ldots \ldots$ and $X_{1}, X_{2}, X_{3}, \ldots$ denote atomic and coarse grained configurational variables respectively, and the corresponding probability distributions are denoted by $P_{\text {all-atom }}\left(x_{1}, x_{2}, x_{3}, \ldots\right)$ and $P_{c g}\left(X_{1}, X_{2}, X_{3}, \ldots\right)$, then we must have

$$
\int F_{\text {all-atom }} P_{\text {all-atom }} d x_{1} d x_{2} d x_{3} \ldots=\int F_{c g} P_{c g} d X_{1} d X_{2} d X_{3} \ldots
$$
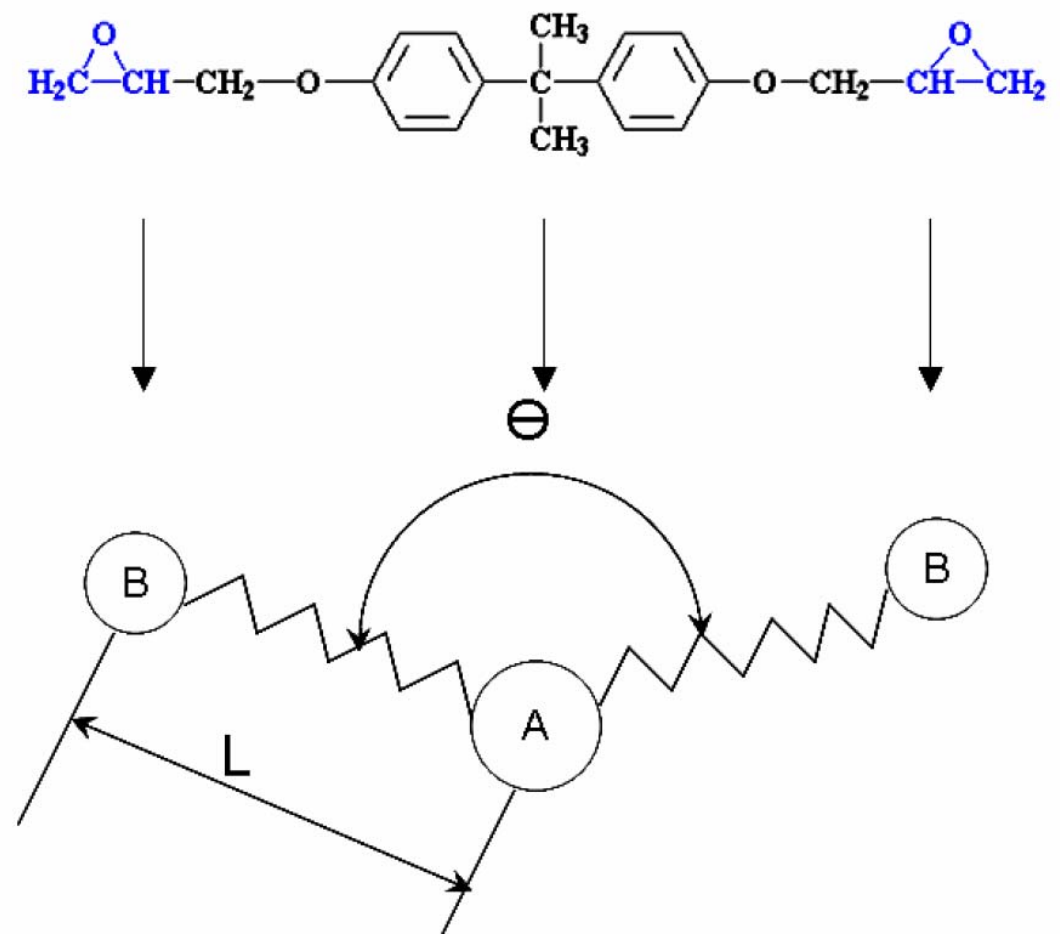

Figure 2: Schematic figure showing the mapping of atomistic model of DGEBA monomer on to the coarse-grained model 


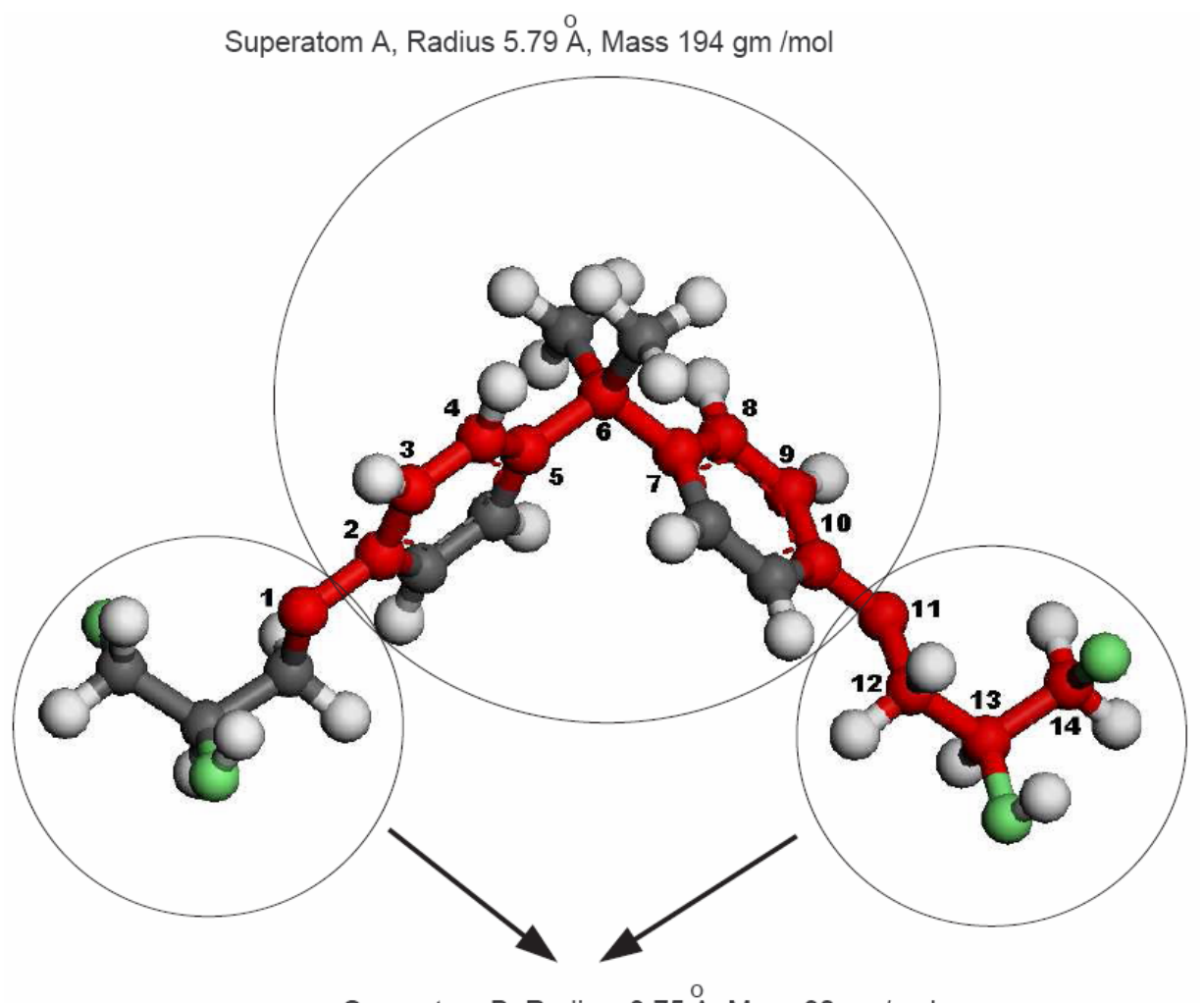

Superatom B, Radius $3.75 \mathrm{~A}$, Mass $90 \mathrm{gm} / \mathrm{mol}$

Figure 3: The optimum geometry of a DGEBA monomer with the masses and radii of the superatoms A and B shown. The atoms marked 1-14 are the ones that are needed to define the whole molecule given that the bond lengths and angles are unchanged.

We may choose bond lengths, bond angles and torsion angles as the atomic variables. We ascribe to all bond lengths and angles their mean values, as if they are fixed. Restricting ourselves to fourth nearest neighbour interactions, the remaining atomistic configuration variables are the torsion angles. It is possible to find the probability distributions of all the torsion angles in the DGEBA molecule. To this end, the DGEBA molecule is mapped onto three superatoms B, A and B as shown in Fig. 2. The geometrical centres of the superatoms coincide approximately with the $\mathrm{C}\left(\mathrm{CH}_{3}\right)_{2}$ and the $\mathrm{CH}(\mathrm{OH})$ groups for superatoms $\mathrm{A}$ and $\mathrm{B}$ respectively. The coarse grained bond length $L$ and bond angle $\Theta$ are also shown in Fig 2. The equilibrium structure of DGEBA and the masses and radii of the superatoms A and B are shown in Fig. 3. The two structures A and B are optimized separately using the commercial software Gaussian. The Hartree-Fock method and the 6-31G(d) basis are used. In view of the fact that all atomic bond angles and lengths are assumed to have fixed values, in the superatoms A and B, there are 14 locations (marked 1-14 in Fig. 3) that have to be determined in order to construct the whole structure. To obtain a torsion potential $V_{i}\left(\phi_{i}\right)$ for the $i$-th torsion angle (i.e. a torsion angle between atoms $i-3, i-2, i-1$ and $i$, we 'measure' the change in the total energy of the structure by varying the angle $\phi_{i}$ keeping all other degrees of freedom fixed at their optimum values. Typical results of this endeavour are shown in Fig. 4(a) through (d) for the torsion angles $\phi_{7}, \phi_{8}, \phi_{13}$, and $\phi_{14}$, the first two belonging to superatom $\mathrm{A}$ and the others to superatom B.

After obtaining all the requisite torsion angles the following steps yield the coarse grained potentials:

1. Several realizations of the DGEBA molecule using the Metropolis Monte Carlo algorithm with the torsion potentials generated are obtained at a chosen temperature $\mathrm{T}=300 \mathrm{~K}$.

2. The positions of the geometrical centres i.e. the positions of the $\left.\mathrm{C}_{(\mathrm{CH}}\right)_{2}$ and $\mathrm{CH}(\mathrm{OH})$ groups are retrieved from the realizations. These are the geometrical centres of the superatoms A and B respectively.

3. The distribution functions $P(L)$ and $P(\Theta)$ for the bond lengths and angles are obtained by plotting histograms as shown in Fig. 5. It should be noted that

$$
\begin{aligned}
& P_{s}(L) \propto \frac{P_{s}^{\prime}(L)}{L^{2}}, \\
& P_{b}(\Theta) \propto \frac{P_{b}^{\prime}(\Theta)}{\sin (\Theta)},
\end{aligned}
$$




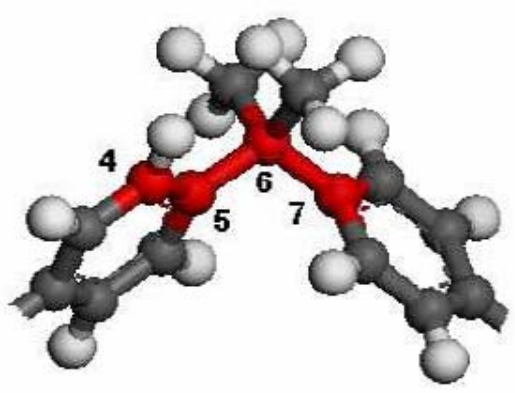

(a)

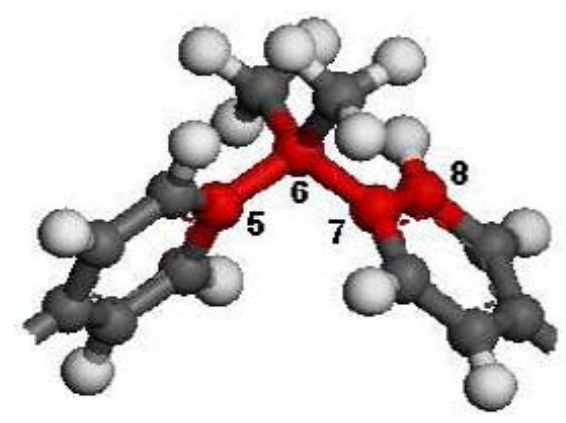

(b)
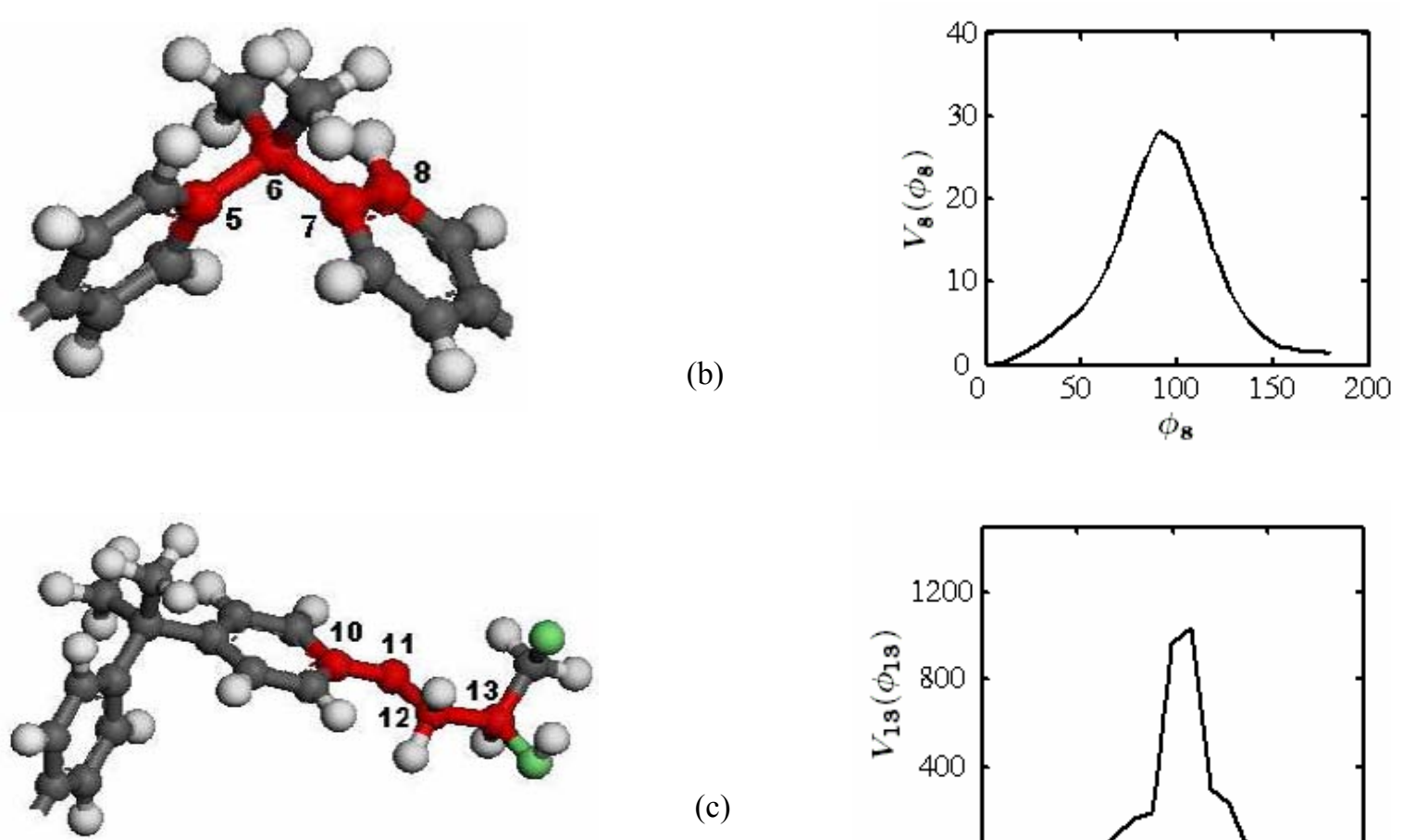

(c)
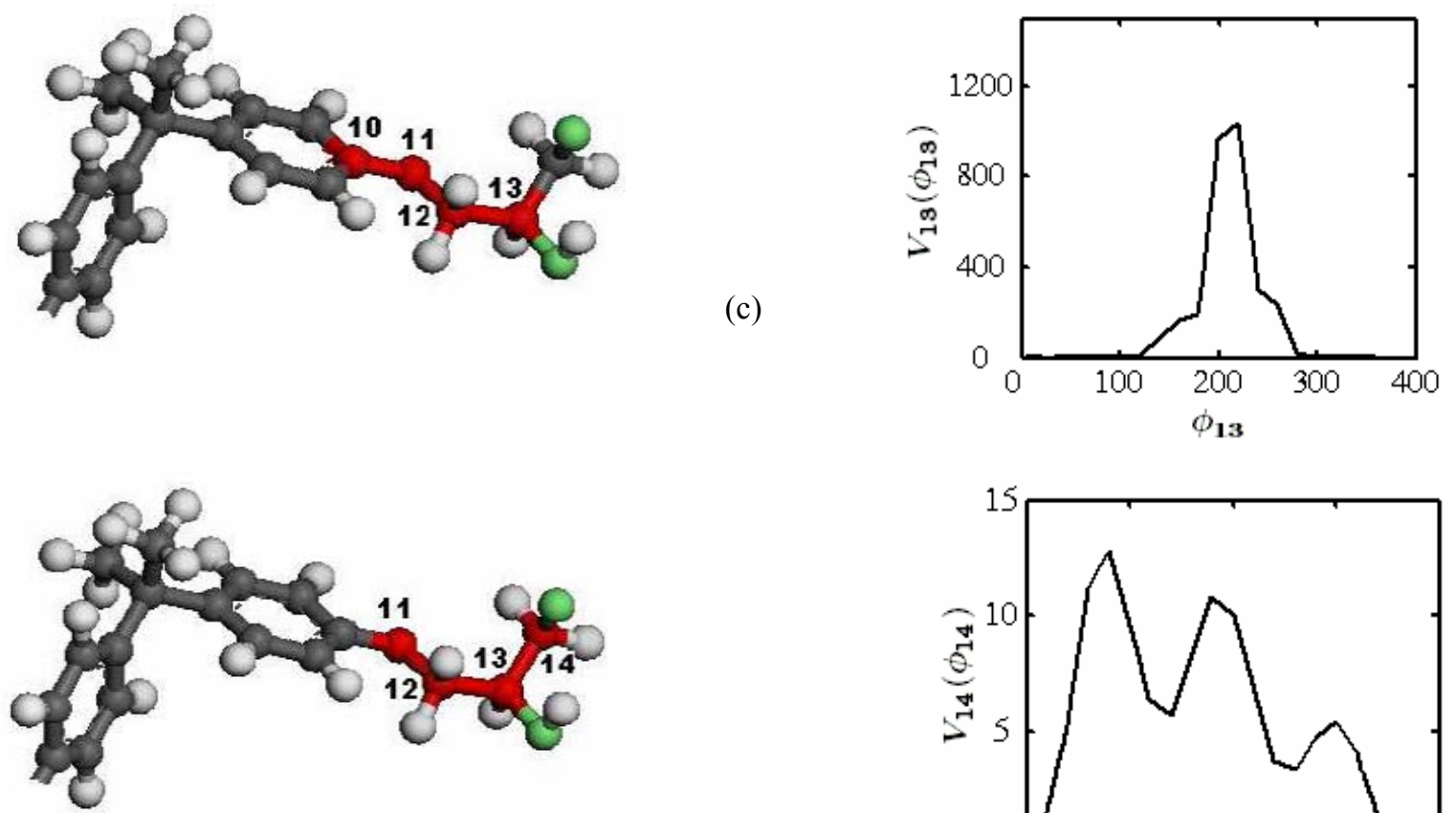
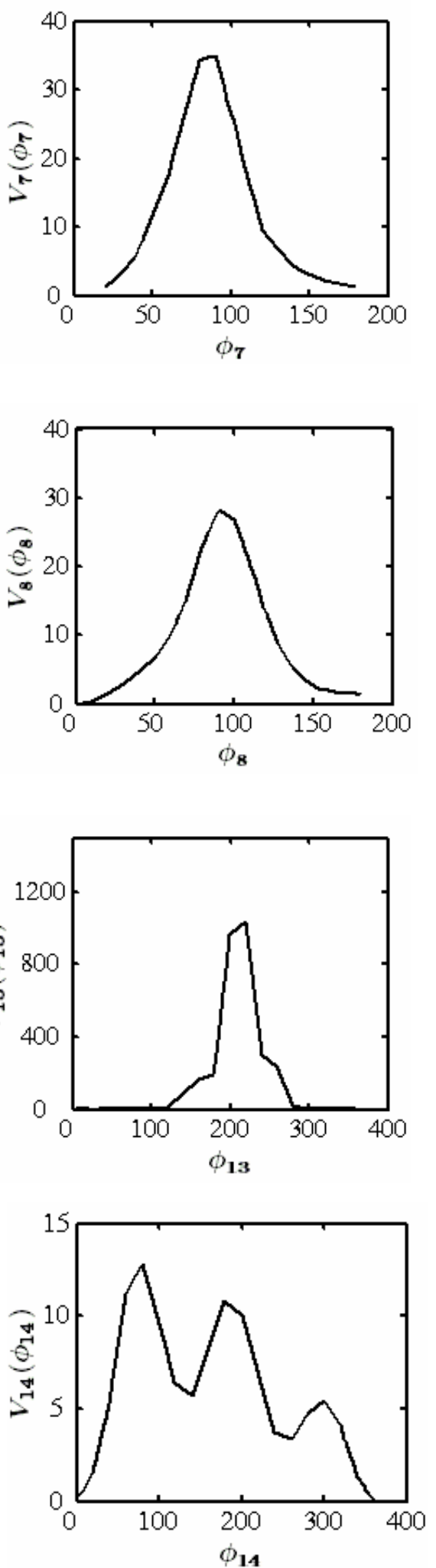

Figure 4: A few representative torsion angles $\varphi_{i}$ and their potentials $V_{i}\left(\varphi_{i}\right)$. In the figure $i=7$ in $(a), i=8$ in $(b), i=13$ in (c) and $\mathrm{i}=14$ in $(\mathrm{d})$.

4. Following Tschop et al. (1998) it is assumed that the coarse-grained potentials factorise i.e.

$$
U(L, \Theta)=U_{s}(L) U_{b}(\Theta),
$$

where, $U_{s}(L)$ and $U_{b}(\Theta)$ are the individual stretching and bending potentials. 


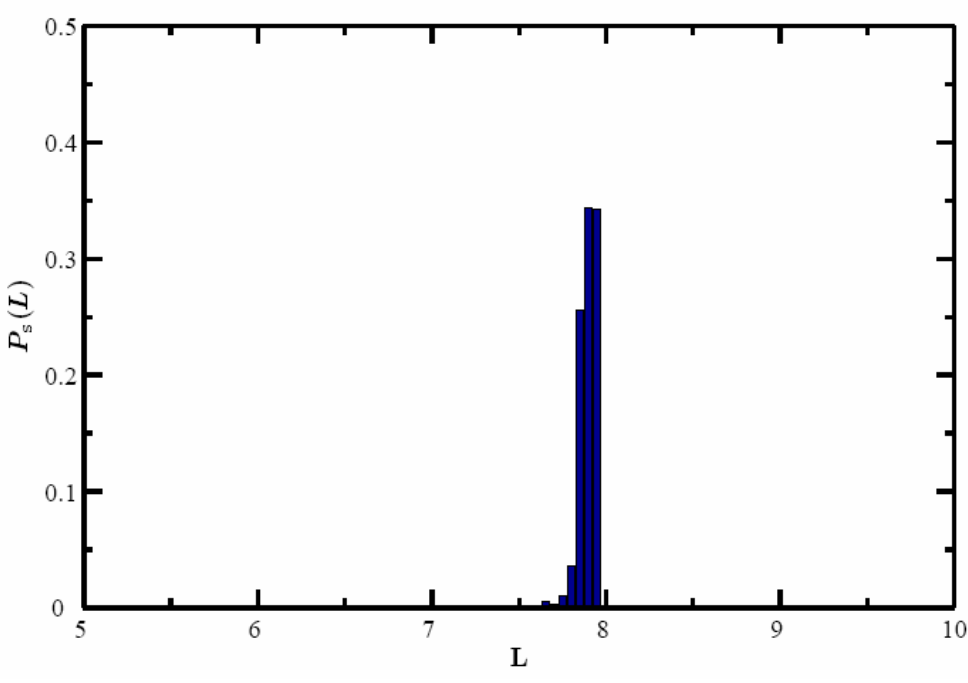

(a)

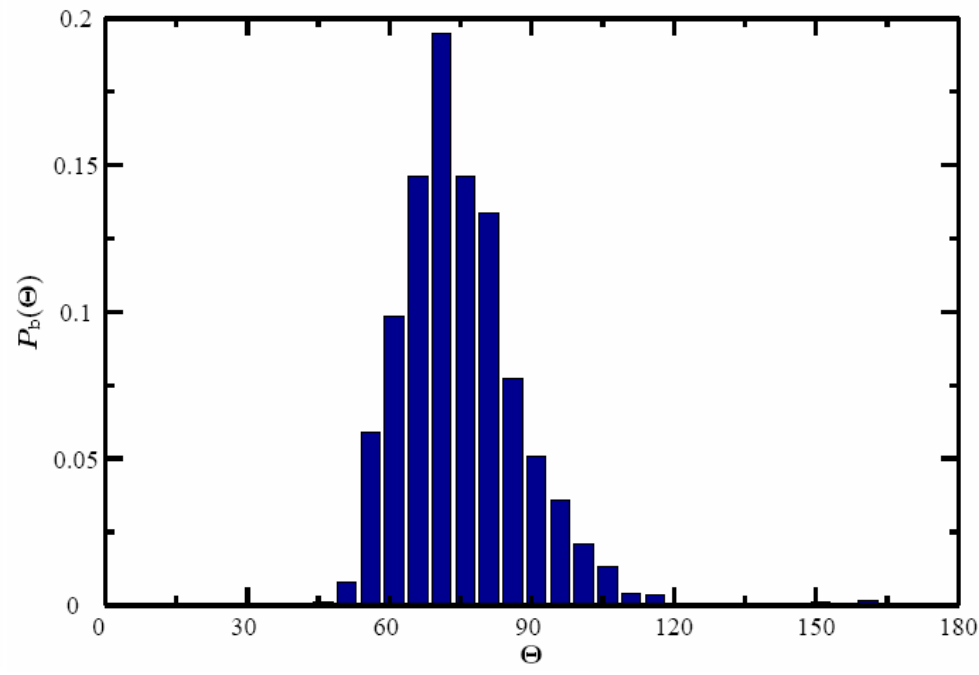

(b)

Figure 5: Histograms obtained from Monte Carlo simulations of the DGEBA structure showing the normalised probabilities of (a) coarse grained stretching and (b) bending.

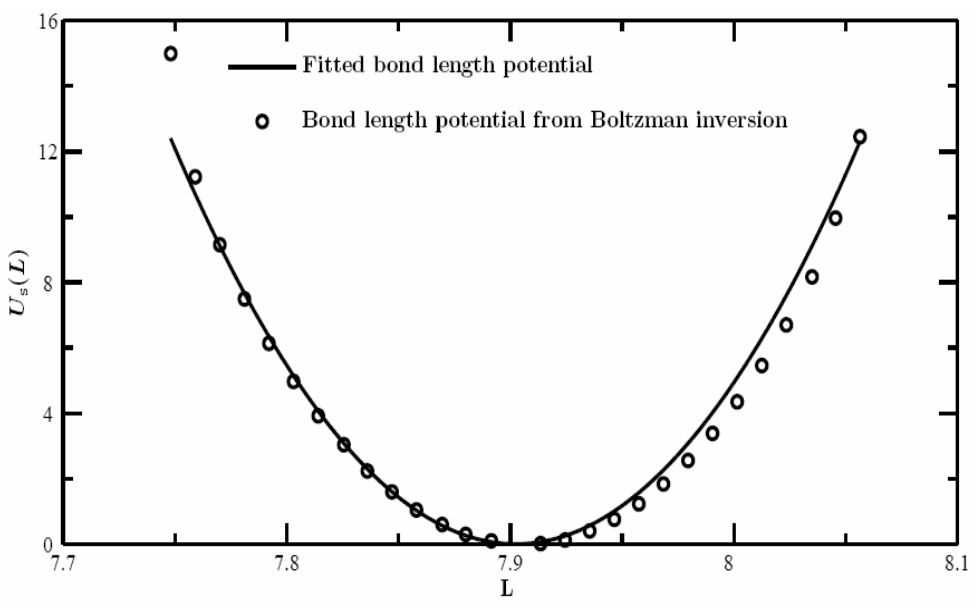

(a)

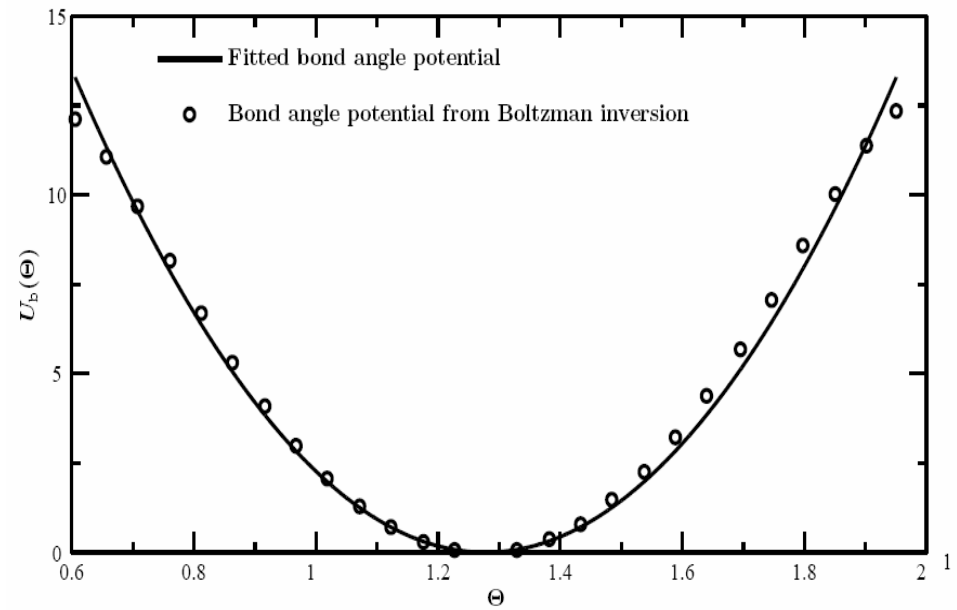

(b)

Figure 6: The fitted bond length (a) and bond angle (b) potential of the coarse-grained model obtained by Boltzmann inversion and fitted with harmonic functions.

This assumption allows us to write the coarse grained potentials by Boltzman inversion as

$$
\begin{aligned}
& U_{s}(L)=-k_{B} T \ln P_{s}(L) \\
& U_{b}(\Theta)=-k_{B} T \ln P_{b}(\Theta) .
\end{aligned}
$$

In the above the Boltzman constant is denoted by $k_{B}$. Figure 6(a) and (b) show the derived potentials for stretching and bending of the coarse grained model shown in Fig. 3. In view of the fact that $P(L)$ and $P(\Theta)$ are, fortuitously, strongly peaked at $L_{0}=7.9 \stackrel{0}{A}$ and $\Theta_{0}=73^{0}$ respectively, harmonic potentials of the forms 


$$
\begin{aligned}
& U_{s}(L)=\frac{1}{2} k_{s}\left(L-L_{0}\right)^{2} \\
& U_{b}(\Theta)=\frac{1}{2} k_{\Theta}\left(\Theta-\Theta_{0}\right)^{2},
\end{aligned}
$$

where, $L_{0}=7.9 \stackrel{0}{A}$ and $\Theta_{0}=73^{\circ}$, fits the Boltzman inverted data quite well. The stiffnesses $k_{s}$ and $k_{\Theta}$ are obtained directly from the fit. The parameters for the L-J potential acting between two superatoms separated by a distance $r$ as,

$$
U_{n b}=4 \in\left[\left(\frac{\sigma}{r}\right)^{6}-\left(\frac{\sigma}{r}\right)^{12}\right],
$$

are obtained by relating the number density $\psi$ to the real density $\rho$ using the formula

$$
\sigma=\left(\frac{\psi M_{m}}{3 \rho}\right)^{1 / 3} .
$$

The factor 3 in the denominator reflects the fact that there are 3 coarse grained superatoms in every DGEBA molecule. The quantity $M_{m}$ is the mass of each monomer in grams. The masses of each superatom are indicated in Fig. 3 . The real density $\rho$ is taken to be $1.17 \mathrm{~g} / \mathrm{cm}^{3}$, which is the experimentally obtained density of uncrosslinked DGEBA at $300 \mathrm{~K}$. The LJ parameter $\sigma$ 0

turns out to be $5.32 \mathrm{~A}$. Following Tschop et al. (1998), we choose $\in=k_{B} T$, whereby $\in=2.5 \mathrm{~kJ} / \mathrm{mol}$. The consequences of this choice are discussed in the appendix of Tschop et al. (1998).

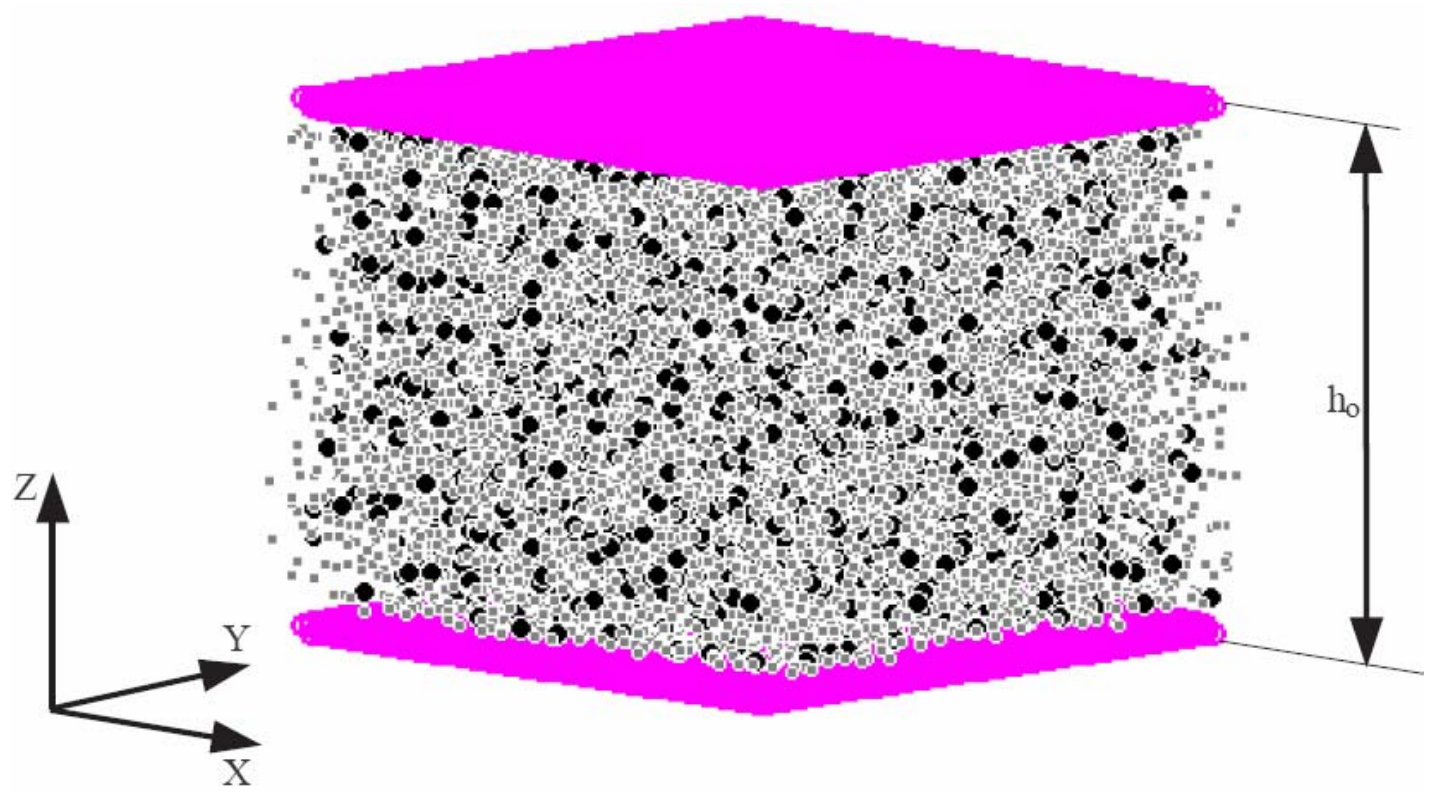

Figure 7: A typical crosslinked sample before pulling. For this sample $\chi=1$ and the sample consists of 1800 four superatom chains and an equal number of three superatom chains with $\mathrm{h} 0=125 \stackrel{\circ}{A}$. 


\section{Crosslinking Strategy}

In this work the diamine crosslinker is trivially coarse grained into one superatom capable of forming four bonds with four DGEBA monomer ends, i.e. the B superatoms in the coarse grained DGEBA molecule. Like Stevens (2001), we start with a simulations box where all crosslinker superatoms have one of the four bonds connected to a coarse grained DGEBA monomer. Optimisation of all the atom structure of the DGEBA molecule with the crosslinker attached shows that the distance between the centres of superatom $\mathrm{B}$ and the crosslinker is about $8 \mathrm{~A}^{0}$.

A sample of coarse grained DGEBA molecules (with three superatoms) and DGEBA molecules attached to a crosslinker (molecules with four superatoms) are placed between two walls. The atoms making up the wall are 'frozen' in the sense that they do not interact with each other and are strongly tethered to their sites by stiff springs. While the non-bonded interaction between the A-A, A-B, B-B, A-crosslinker and B-crosslinker superatoms are governed by the same LJ parameters, the interaction energy $\epsilon_{w}$ between all superatoms and the atoms comprising the wall is taken to be $3 \in$. The parameter $\epsilon_{w}$ governs the strength of the interface between the adhesive and adherend. By taking $\epsilon_{w}=3 \in$, a value arrived by trial and error, we have made adhesive failure along the interface relatively difficult.

All samples are generated such that there are as many three superatom molecules as there are four superatom molecules to ensure that complete crosslinking can happen. The initial sample was first equilibrated under NVE control till the total energy becomes constant. This was followed by equilibration under NVT at $300 \mathrm{~K}$ and was followed by NPT equilibration at the same temperature and 1 bar pressure. After attaining the desired temperature and pressure, the crosslinking process was started and carried out under NPT control. In the crosslinking process a new bond was formed between the end of a coarse grained DGEBA molecule (superatom B) and the unoccupied bond of a crosslinker when the distance between a superatom B and a crosslinker bead was less than $8 \stackrel{0}{A}$.

The energy of the system was calculated taking the bond energies of the new bonds formed. A typical crosslinked sample is shown in Fig. 7 where the small light coloured circles represent the crosslinker superatoms.

As crosslinking proceeds, the density of the system quickly attains a constant value of about $1.38 \mathrm{gm} / \mathrm{cc}$, which is slightly above the experimentally measured density of crosslinked DGEBA. This discrepancy is a consequence of the value of $\rho$ we have assumed while computing $\sigma$ in eq. 4 which pertains to the uncrosslinked sample. The variation of the density $\rho_{\text {actual }}$ with time, for a typical crosslinking simulation is shown in Fig. 8.

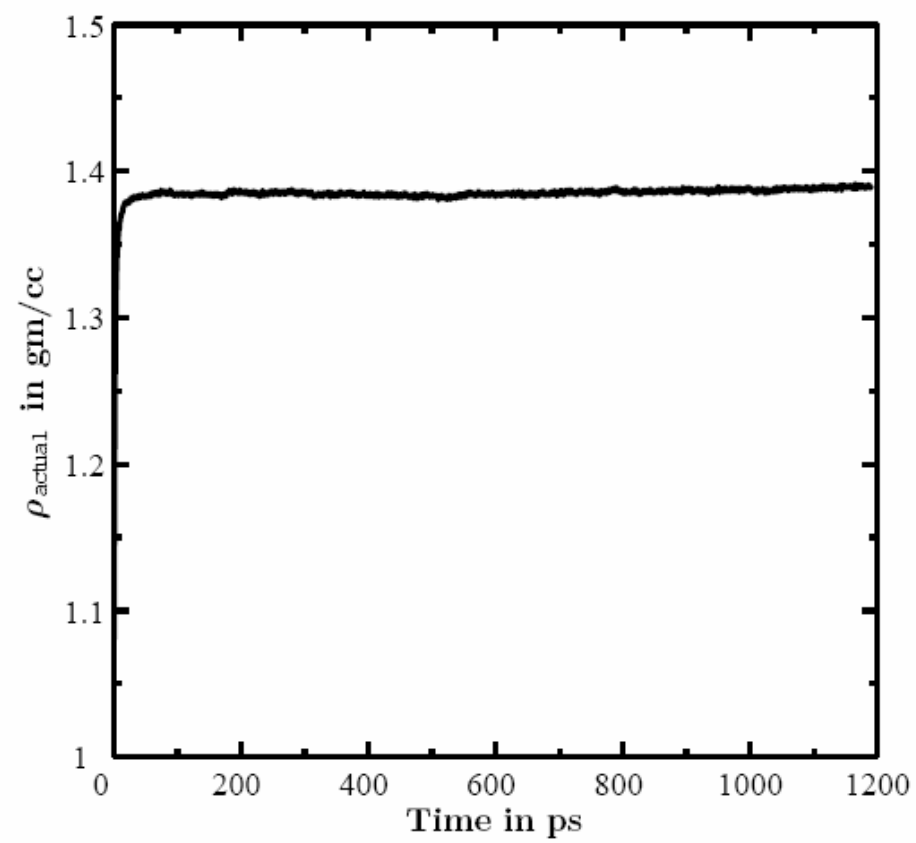

Figure 8. The variation of the actual density $\rho_{\text {actual }}$ with time during crosslinking. 


\section{Determination of the energy of adhesion}

In the final part of the analyses, we attempt to determine the energy per unit surface area required to separate the crosslinked confined layer completely. To this end, the rigid layers at the top and bottom are pulled apart at a velocity of $0.005 \tau^{-1}$ where $\tau$ is the simulation timestep. In all the simulations reported hereafter, $\tau$ is $5 \mathrm{fs}$. The pulling is done under NVT control within each timestep, keeping the area perpendicular to $z$ axis fixed and periodic boundary conditions applied in the $x$ and $y$ directions. The boundary conditions applied simulate an infinite slab of crosslinked DGEBA of an initial thickness $h_{0}$.

Calculation of stress uses the equation

$$
\Sigma^{\alpha \beta}=\frac{1}{2 \Omega} \sum_{i=1}^{N_{c} n_{c}} \sum_{j \neq i} r_{j i}^{\alpha} f_{j i}^{\beta},
$$

where $\Omega$ is the volume over which the stress is averaged. Unless otherwise mentioned, this volume is of the whole system. The $\alpha$ component of the distance vector between atoms $j$ and $i$ is denoted by $r_{j i}^{\alpha}$ (not to be confused with contravariant components) and the $\beta$ component of the force exerted on $j$ by $i$ is given by $f_{j i}^{\beta}$. The above definition of the atomic stress eq.5 has been shown to be equivalent to the Cauchy stress tensor $\left\langle\sigma^{\alpha \beta}\right\rangle$ averaged over the volume $\Omega$ (Zhou, 2003; Chen and Fish, 2006).

The total energy of the system is the sum of the non-bonded energy $U_{n b}$, the total energy due to stretching of bonds $U_{s}$ and that due to bond bending $U_{b}$. The force component $f_{j i}^{\beta}$ is determined for the non-bonded and stretching interactions as

$$
f_{j i}^{\beta}=-\frac{\partial U_{p}}{\partial r_{j i}^{\alpha}},
$$

where, $p=n b$ or $s$. The contribution to the stress $\Sigma^{\alpha \beta}$ from bond bending for a set of consecutive atoms $i, j$ and $k$ is,

$$
r_{i j}^{\alpha} f_{j}^{\beta}+r_{i k}^{\alpha} f_{k}^{\beta}
$$

where

$$
f_{i}^{\alpha}=-\frac{\partial U_{b}}{\partial r_{i}^{\alpha}}
$$

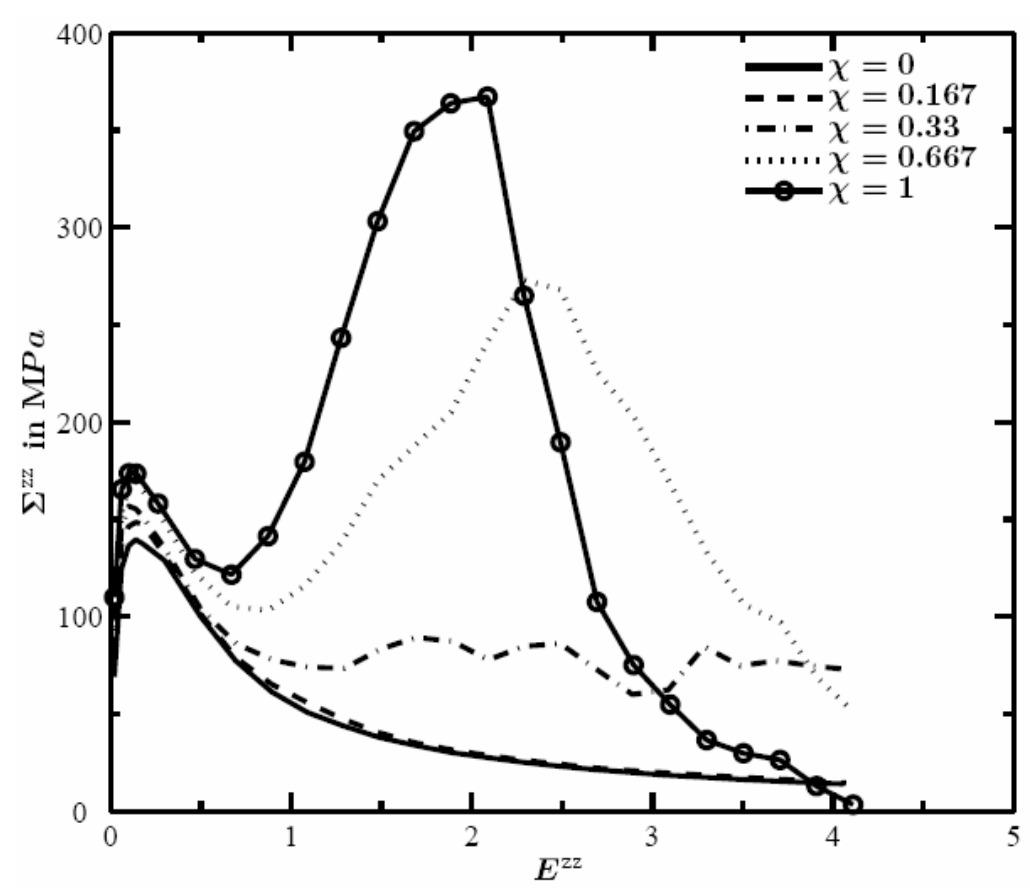

Figure 9. Variation of the viral stress $\sum^{\mathrm{zz}}$ in $\mathrm{MPa}$ with strain at different values of $\chi$. 


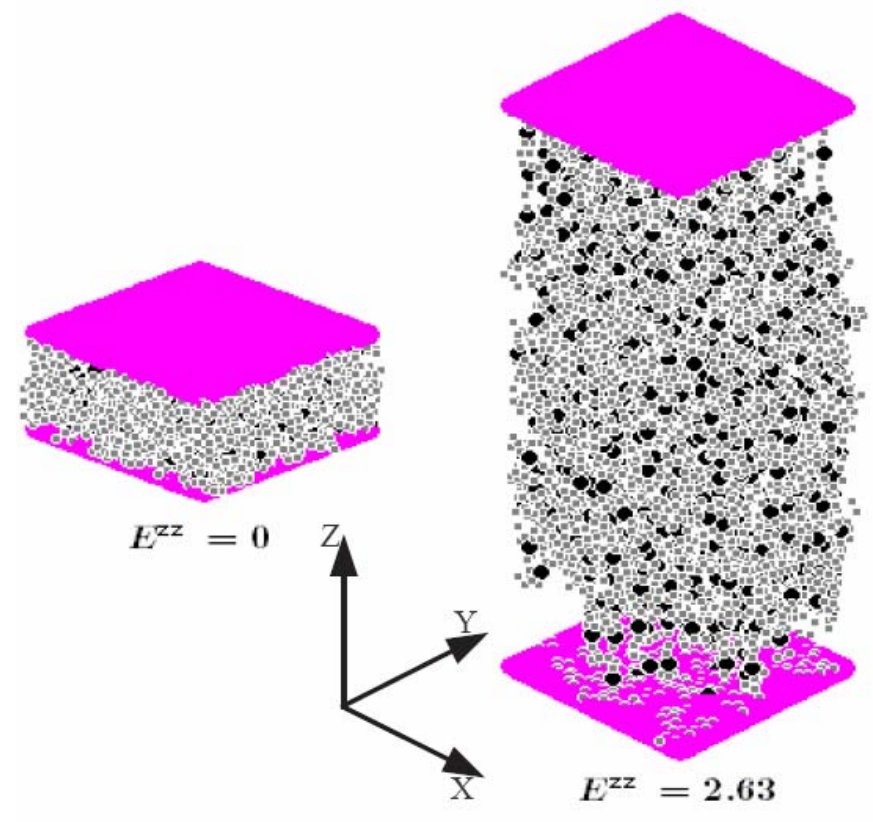

(a)

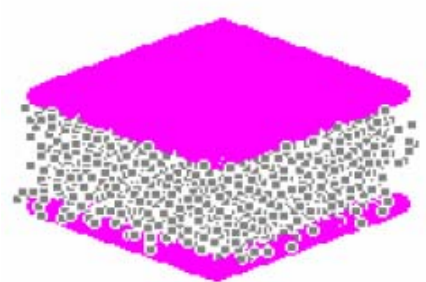

$E^{z z}=0$

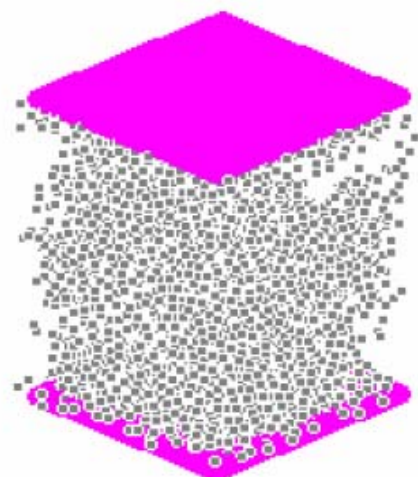

$E^{z z}=0.99$

(b)
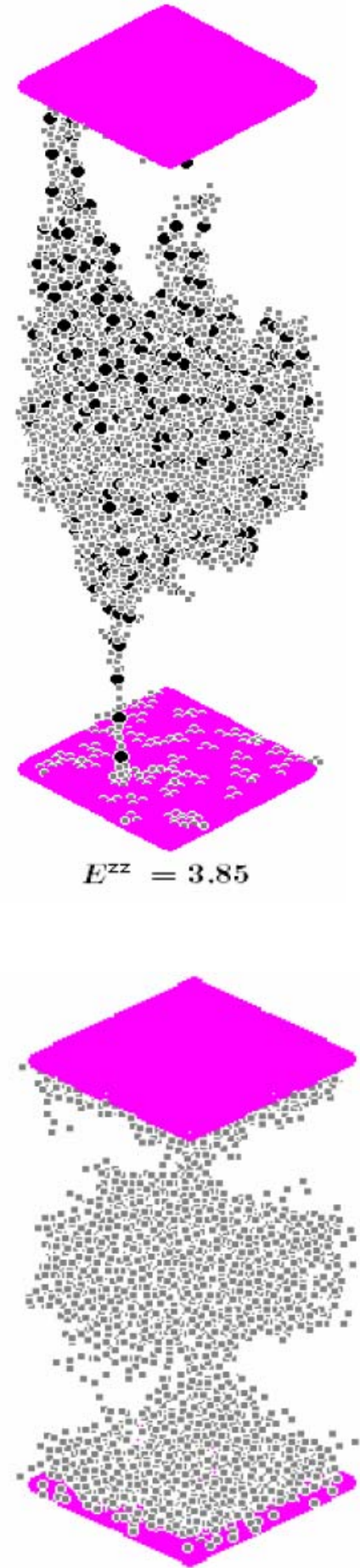

$E^{\mathrm{zz}}=1.78$

Figure 10. Deformed configurations of two samples with (a) $\chi=1$ and (b) $\chi=0$ at the overall values of strain indicated in the figure. 
A quantity $\chi$ was defined as the ratio of the number of crosslinker superatoms to the number of three superatom chains used. Thus, this quantity is indicative of the degree of crosslinking in the sample. The stress strain response of samples (where strain at time $t$ is simply defined as $\mathrm{E}^{z z}(t)=h(t) / h_{0}-1, h$ being the height of the sample at time $t$ ) with varying $\chi$ are shown in Fig. 9. When $\chi=0$, no crosslinking exists and the DGEBA molecules are unconnected. The stress carrying capacity of such a sample, as shown in Fig. 10 is very low, governed only by the non-bonded interactions between the DGEBA molecules. The stress strain response shows a maximum after which, the stress drops to zero, indicating quick and complete separation.

On the contrary, at high values of $\chi$, the DGEBA monomers form networks through the crosslinkers. At high degrees of crosslinking continuous pathways spanning the thickness of the layer are established. The interconnectivity introduced by crosslinking, as also noted by Stevens (2001), renders the layers stronger and more ductile. The overall stress carrying capacity also increases with $\chi$. More importantly, as $\chi$ increases, the samples deform significantly before separating. Highly crosslinked samples also soften after reaching the maximum stress at the end of the elastic part of the stress strain curve. The samples harden thereafter but the amount of hardening is a function of $\chi$. For $\chi \rightarrow 1$, the softening and subsequent hardening response is quite like that of long chained entangled glassy polymers. The degree of crosslinking however, does not affect the elastic modulus which is about $1.37 \mathrm{GPa}$.

Figure 10(a) and (b) shows the deformed configurations of two samples with $\chi=1$ and $\chi=0$ respectively at three stages of deformation. For the sample with $\chi=0$, the failure ensues at a void that has nucleated in the bulk of the sample. With stretching, this void spreads sideways to form the separating surfaces. On the contrary, owing to the increased cohesive strength by virtue of the crosslinks, in the sample with higher $\chi$ the voids appear at the interface with the walls rather than in the bulk. Failure happens due to the growth of these interfacial voids and when complete separation occurs, it does close to the interface.

It is instructive at this stage to compare the energy of separation obtained from our MD computations with continuum fracture mechanics results. In the MD simulations, the energy per unit undeformed surface area required to cause complete separation is calculated as

$$
W=h_{0} \int_{0}^{E_{\text {final }}^{z z}} \Sigma^{z z} d \mathrm{E}^{z z}
$$

where $\mathrm{E}_{\text {final }}^{z z}$ is the strain at failure. In this relation we have assumed equivalence between the volume average of the Cauchy stress and the virial stress defined in eq. 5 based on the whole volume of the system.

A linear elastic fracture mechanics (LEFM) plane strain solution to the debonding of an adhesively bonded butt joint with rigid adherends has been proposed by Reedy (2002). In this analysis it is assumed that fracture ensues from a corner crack of initial length $a$ at the interface and quickly causes separation at the interface when the energy release rate $\varsigma$ exceeds $\zeta_{c}$. The stress intensity factor $\mathrm{K}$ in the case of a corner crack exhibits a singularity of the order $1-\lambda$ where $\lambda \square 0.7$. Thus the energy release rate is given by

$$
\varsigma=\frac{\left(1-\beta^{2}\right)}{\mathrm{E}^{*}} K_{a}^{2} a^{2 \lambda-1} D(1, \beta)
$$

In the above equation, $\beta$ is a Dundur's parameter for a rigid-elastic interface, $\mathrm{E}^{*}=\frac{2 \mathrm{E}}{\left(1-v^{2}\right)}, \mathrm{E}$ is the Young's modulus, $v$ the Poisson's ratio and $\mathrm{D}$ is a parameter dependent on the elastic mismatch.

Using the above idea of the interface fracture ensuing from a corner crack, Reedy (2002) conducted experiments on butt joints of steel adherends and DGEBA based adhesives to show that a thickness $h_{0}$ independent value of $K_{a}=K_{a c}$ at which rapid crack propagation starts can be found. Note that the thickness independence is in the range of macroscopic thickness and an initial corner flaw of macroscopic dimensions was deliberately introduced in the experiments. The value of $K_{a c}$ turns out to be around 13 $\mathrm{MPamm}^{0.3}$ for DGEBA adhering to steel (almost rigid with respect to epoxy). Using $\mathrm{E}=1.37 \mathrm{GPa}, v=0.35, \beta=0.231$, $D(1, \beta)=5.89$ and $a / h_{0}=0.1$, the value of $\varsigma_{c}$ turns out to be $1.3 \mathrm{~J} / \mathrm{m}^{2}$. It would be higher for smaller values of $a / h_{0}$. 
The LEFM based value of $\zeta_{c}$ is plotted along with the computed values of $W$ from eq. 9 in Fig. 11. Clearly LEFM underpredicts the value of the separation energy through the values of $W$ shown represents the energy expended to separate perfectly bonded materials and not precracked ones. Moreover, for the cases with high values of $\chi$, where interfacial voiding causes separation, LEFM is a poor approximation of reality as clearly the amount of plastic deformation is significant. Experimental values of adhesion energy between epoxy and relatively rigid silicon obtained through other methods (see Hohlfelder 1998) range between 2 and $25 \mathrm{~J} / \mathrm{m}^{2}$. In view of the scatter in experimental estimates, our value of around $8 \mathrm{~J} / \mathrm{m}^{2}$ does not seem very unrealistic. Also important is the fact that the stress carrying capacity of the adhesive increases with crosslinking. The maximum stresses borne by the layer as an almost linear function of $\chi$ is also shown in Fig. 11.

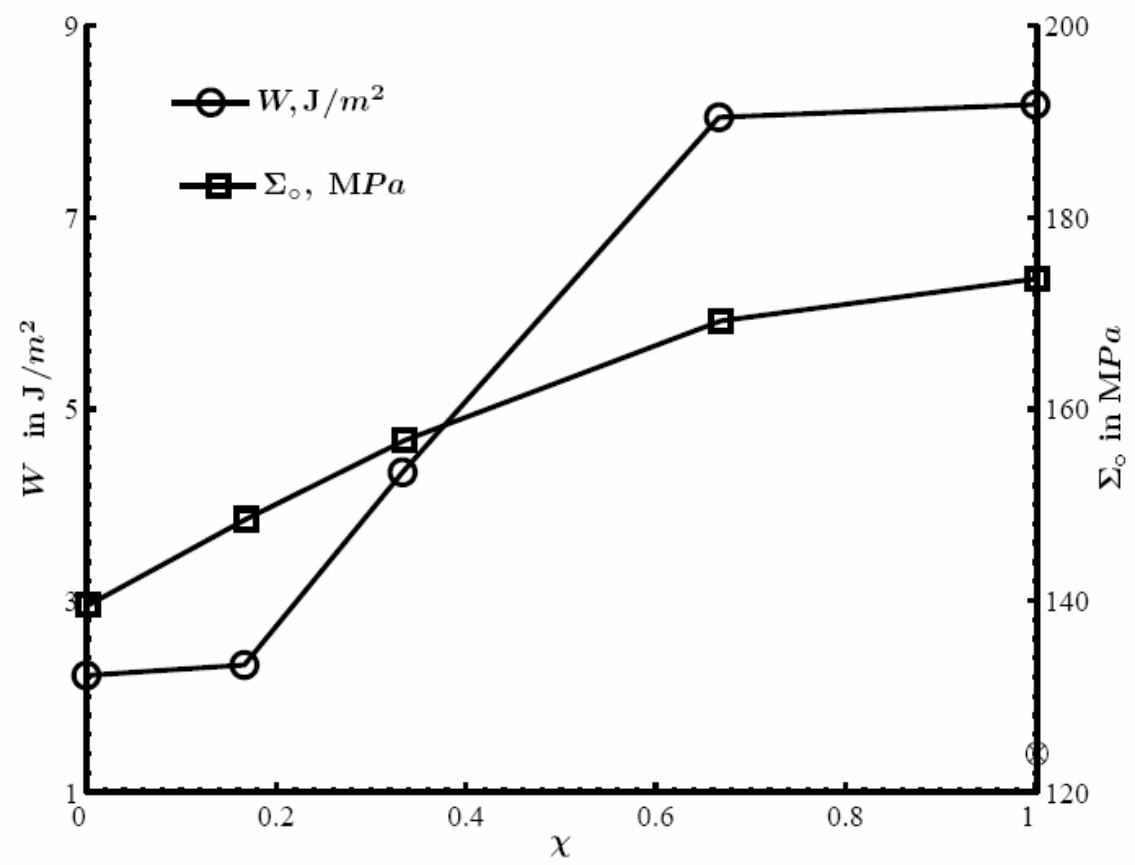

Figure 11. Variation of the adhesion energy $\mathrm{W}$ and the yield $\sum_{\mathrm{o}}$ with the degree of crosslinking $\chi$. The $\otimes$ at the bottom right indicates the separation energy for a butt joint obtained from a LEFM estimate for a fully cured DGEBA layer with a/h $\mathrm{h}_{0}=0.1$.

\section{Summary and Conclusions}

In this work, we have been able to predict, to the best of our knowledge for the first time, the work of separation of a commonly used commercial adhesive system starting merely from its chemical architecture and a simple description of the crosslinking process. This is an important step towards applying coarse grained MD simulation techniques to the design of polymeric adhesives for various applications.

The process starts with a coarse graining strategy wherein the detailed molecular architecture is simplified to conveniently chosen superatoms. The crux of the coarse graining process is in determining the interaction potentials, both bonded and nonbonded, between the superatoms. This is followed by a simulation of the crosslinking between the coarse grained molecules and the coarse grained crosslinkers. Finally, the crosslinked sample is pulled under tension to complete separation and the work of separation is determined from the area under the virial stress strain curve. The values of the work of separation per unit undeformed area fall within the degree of scatter seen in experimental results.

As a final word of caution we wish to point out that the success of such predictions depend to a large extent on the deformation rate dependence of the property being sought. As MD is performed at unrealistically high rates of deformation, rate dependent properties tend to be overestimated. For a few cases reported in this work we have also performed simulations at rates 10 times slower than what is described above. It is seen that for the material analysed $W$ is somewhat weakly dependent on rate. Similarly, the effect of the size scale $h_{0}$ has also been studied and the results reported are almost invariant with $h_{0}$ chosen. It will be interesting to see if further coarsening of the superatoms is possible keeping the quality of the predictions intact. 


\section{Acknowledgement}

One of the authors (SB) gratefully acknowledges financial support received from the Department of Science and Technology, Government of India through project number SR/FTP/ETA-31/2002 and from the Indian Space Research Organisation through project number ISRO/STC/ME/2003090.

\section{References}

Akkermans, R.L.C. and Briels, W.J., 2001. A structure-based coarse-grained model for polymer melts. Journal of Chemical Physics. Vol. 114, pp. 1020-1031.

Baschnagel, J. et al, 2000. Bridging the gap between atomistic and coarse-grained models of polymers: status and perspectives. Advances in Polymer Science. Vol. 152, 41.

Brown,D. and Clarke, J.H.R., 1991. Molecular dynamics simulation of an amorphous polymer under tension. 1. Phenomenology. Macromolecules. Vol. 24, pp. 2075-2082.

Bulacu, M. and Van der Geissen, E., 2005. Effect of bending and torsion rigidity on self-diffusion in polymer melts: a molecular dynamics study. Journal of Chemical Physics. Vol. 123, 114901-1-13.

Capaldi, F.M., Boyce, M.C. and Rutledge, G.C., 2002. Enhanced mobility accompanies the active deformation of a glassy amorphous polymer. Physical Review Letters. Vol. 89, pp. 175505/1-175505/4.

Chen, W. and Fish, J., 2006. A generalized space-time mathematical homogenization theory for bridging atomistic and continuum scales. International Journal for Numerical Methods in Engineering. Vol. 67, pp. 253-271.

Ikegami, K., Fujii, T., Kawagoe, H., Kyogoku, H., Motoie, K., Nohno, K., Sugibayashi, T. and Yoshida, F., 1996. Benchmark tests on adhesive strengths in butt, single and double lap joints and double-cantilever beams. International Journal of Adhesion and Adhesives. Vol. 16, pp. 219-226.

Kremer, K. and Grest, G., 1995. Computer simulations in polymer physics. Physics World. Vol. 8, pp. 39-44.

Kulmi, U. and Basu, S., 2006. A molecular dynamics study of the failure modes of a glassy polymer confined between rigid walls. Modelling and simulation in Materials Science and Engineering. Vol. 14, pp. 1071-1093.

Lyulin, A.V. and Michels, M.A.J., 2006. Simulation of polymer glasses: From segmental dynamics to bulk mechanics. Journal of Non-crystalline Solids. Vol. 352, pp. 5008-5012.

McCoy, John D. and Curro, John G., 1998. Mapping of explicit atom onto united atom potentials. Macromolecules. Vol. 31, pp. 9362-9368.

Mondello, M., Yang, H., Furuya, H. and Roe, R., 1994. Molecular dynamics simulation of atactic polystyrene 1. Comparison with X-ray scattering data. Macromolecules. Vol. 27, pp. 3566-3574.

Muller-plathe, F., 2002. Coarse-graining in polymer simulation: From the atomistic to mesoscopic scale and back. ChemPhysChem, Vol. 3, p. 754.

Negi, A. and Basu., 2006. A molecular dynamics study on the strength and ductility of high Tg polymers. Modelling and Simulation in Materials Science and Engineering. Vol. 14, pp. 563-580.

Ogura, I. and Yamamoto, T., 1995. Molecular dynamics simulation of large deformation in an amorphous polymer. Polymer. Vol. 36, pp. 1375-1381.

Reedy, E.D, Jr., 2002. in Adhesion Science and Engineering-I: The Mechanics of Adhesion. eds. Dillard, D.A. and Pocius, A.V., Amsterdam (Elsevier Science), pp. 145-192.

Rottler, J. and Robbins, M.O., 2003. Molecular simulations of deformation and failure in bonds formed by glassy polymer adhesives. Journal of Adhesion Science and Technology. Vol. 17, pp. 369-381.

Steele, D., 1985. An ab initio investigation of the torsional potential function of n-butane. Journal of the Chemical Society Faraday Transactions II. Vol. 81, pp. 1077-1083.

Stevens, M.J., 2001. Interfacial fracture between highly cross-linked polymer networks and a solid surface: Effect of interfacial bond density. Macromolecules. Vol. 34, pp. 2710-2718.

Sun, Q. and Faller, R., 2005. Systematic coarse-graining of atomistic models for simulation of polymeric systems. Computers \& Chemical Engineering. Vol. 29, pp. 2380-2385.

Theodorou, D.N., 2004. Understanding and predicting structure property relations in polymeric materials through molecular simulations. Molecular Physics. Vol.102, pp. 147-166.

Tschop, W., Kremer, K., Batoulis, J., Burger, T. and Hahn, O., 1998. Simulation of polymer melts. I Coarse-graining procedure for polycarbonates. Acta Polymer. Vol. 49, pp. 61-74.

Weiner, J.H. and Gao, J., 1994. Simulation of viscoelasticity in polymer melts: Effect of torsion potential. Modelling and Simulation in Materials Science and Engineering. Vol. 2, pp. 755-766.

Yashiro, K., Ito, T. and Tomita, Y., 2003. Molecular dynamics simulation of deformation behaviour in amorphous polymer: Nucleation of chain entanglements and network structure under uniaxial tension. International Journal of Mechanical Sciences. Vol. 45, pp. 1863-1876.

Zhou, M., 2003. A new look at the atomic level virial stress: on continuum-molecular system equivalence. Proc. R. Soc. Lond. A. Vol. 459, pp. 2347-2392. 
Biographical notes:

Sumit Basu did his PhD with Prof R Narasimhan from the Indian Institute of Science Bangalore. After a four year post doctoral stint with Prof Erik van der Giessen, working on various aspects of the micromechanics of materials (especially polymers), he joined the Indian Institute of Technology Kanpur, where he is now an Associate Professor in the Department of Mechanical Engineering. His research involves studying, mainly through computational means, the micromechanical aspects of deformation and failure in materials.

Tarun Grover is a graduate student at the Massachusetts Institute of Technology, USA in the Department of Physics. He got his undergraduate degree from the Indian Institute of Technology Kanpur.

Siva Prasad AVS currently doing PhD in the Department of Mechanical Engineering, IIT Kanpur. He did his B.Tech from Sri Venkateswara University College of Engineering, India from 2001 to 2005. He then did his M.Tech with Dr. Sumit Basu at IIT Kanpur from 2005 to 2007. He then joined for PhD at IIT Kanpur in 2008 after one year of industrial experience.

Received November 2009

Accepted March 2010

Final acceptance in revised form May 2010 